\title{
Princicpal Component Analysis of type II supernova $V$ band light-curves
}

\author{
Lluís Galbany ${ }^{1,2}$ \\ ${ }^{1}$ Millennium Institute of Astrophysics, Universidad de Chile, \\ Casilla 36-D, Santiago, Chile \\ ${ }^{2}$ Departamento de Astronomía, Universidad de Chile, \\ Casilla 36-D, Santiago, Chile \\ email: lgalbany@das.uchile.cl
}

\begin{abstract}
We present a Principal Component Analysis (PCA) of the $V$ band light-curves of a sample of more than 100 nearby Core collapse supernovae (CC SNe) from Anderson et al. (2014). We used different reference epochs in order to extract the common properties of these light-curves and searched for correlations to some physical parameters such as the burning of ${ }^{56} \mathrm{Ni}$, and morphological light-curve parameters such as the length of the plateau, the stretch of the light-curve, and the decrements in brightness after maximum and after the plateau. We also used these similarities to create SNe II light-curve templates that will be used in the future for standardize these objects and determine cosmological distances.
\end{abstract}

Keywords. techniques: photometric, methods: statistical, (stars:) supernovae: general

\section{Introduction}

SNe II represent the most homogeneous set of CC SNe and, although they are on average $\sim 1.2 \mathrm{mag}$ intrinsically fainter than SNe Ia, their use as independent cosmological distance indicators has been already demonstrated in Hamuy \& Pinto (2002). They are also the most common of all SN types, although there is a deficit in the literature as compared to the prioritized SNe Ia, especially at higher redshift. The advantages of SNe II as cosmological probes over SNe Ia reside on the simplicity of their hydrogen dominated atmospheres and low-density surrounding media, and on the current understanding of the explosion mechanism and progenitor system. These advantages together with their high rates and homogeneity, make SNe II promising independent cosmological probes.

\section{Analysis}

We have used a sample of 116 low-redshift SNe II from Anderson et al. (2014) for which several $V$ band light-curve parameters have been determined and measured. This parameters include absolute magnitudes at the peak, at the end of the plateau, and at the beginning of the radioactive decline. There are also determined the explosion epoch and the epochs when the plateau starts and ends, from which the duration of the plateau and of the optically thick phase can be measured. Other parameters such as the amount of ${ }^{56} \mathrm{Ni}$ burned, and the three decrements in magnitude brightness from the peak to the transition, during the plateau, and during the radioactive phase, are also measured from the SN light-curves. The PCA required an optimal selection of the epochs available to both (i) have a wide range that allows a compete standardization of the light curves, and (ii) keep a significant number of SNe with observed data in the range previously selected. The selection of a time reference and a normalization parameter was a relevant 

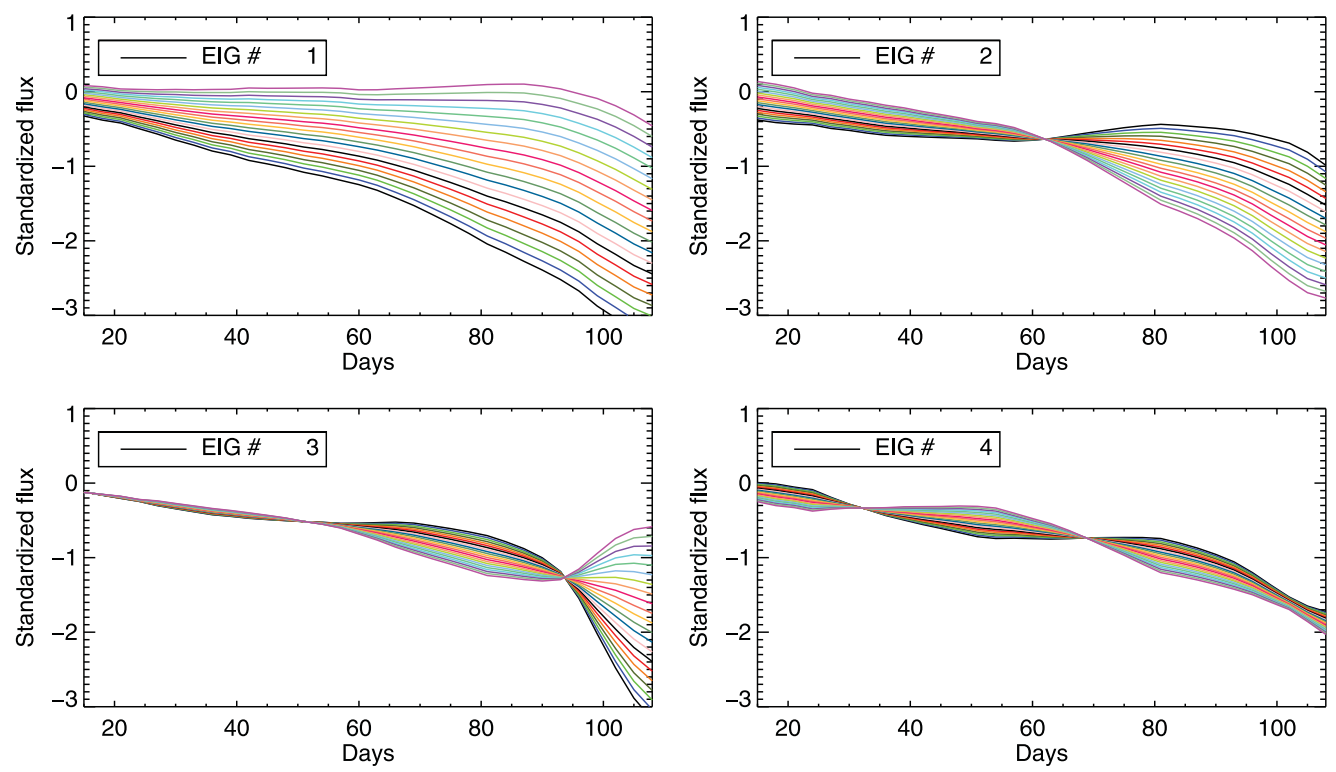

Figure 1. First four eignevectors of the PCA. The variation of the eigenvector when multiplied by different projections is showed in difference colors. The range of projections applied is selected from the $2 \sigma$ variation from the mean in our sample.

determination as well, since not all the light-curve parameters are available for all the SNe in this sample. After several attempts the best approach was to normalize the lightcurves in brightness to the absolute magnitude at the peak and use the explosion date as the reference epoch. Once this was established, we selected all the light-curves with observations before +15 days and after +110 days from the explosion, and applied a linear interpolation in steps of three days in order to have all the light-curves with the same temporal cadence. This resulted in a sample of 29 SNe.

Finally, we have performed a PCA of the $29 \times 32$ matrix containing the 32 epochs for each of the $29 \mathrm{SNe}$. The PCA gave an orthonormal set of 32 eigenvectors (EV) ordered by decreasing variability, and a matrix of 29x32 projections (one projection for SN and EV). In our case, the first four EVs accounted for more than the $99 \%$ of the light-curve variation. In Figure 1, one can see the contribution of this four EV to reproduce a SN light-curve by multiplying the 2-sigma variation of all 29 SN projections in each of these 4 EVs.

\section{Results}

We have correlated each pair of projection and physical or light-curve parameter for all SNe in our sample for which the required information was available. Strong correlations between each projection with different light-curve parameters are found.

- The projections of the first EV correlates to the absolute peak magnitude $\left(M_{\max }\right)$, and the decline rates from the peak the the plateau $(s 1)$, and during the plateau $(s 2)$. In Figure 2 one can see the correlation between these projections and the s 2 parameters.

- The second EV correlates with the duration of the plateau $\left(t_{P T}\right)$, which is related to the total amount of Hydrogen present in the outer layers of the SNe.

- The projections of EV3 shows the best correlation with the total amount of ${ }^{56} \mathrm{Ni}$ mass synthesized by the $\mathrm{SN}$, which is measured in the radioactive decline phase. 


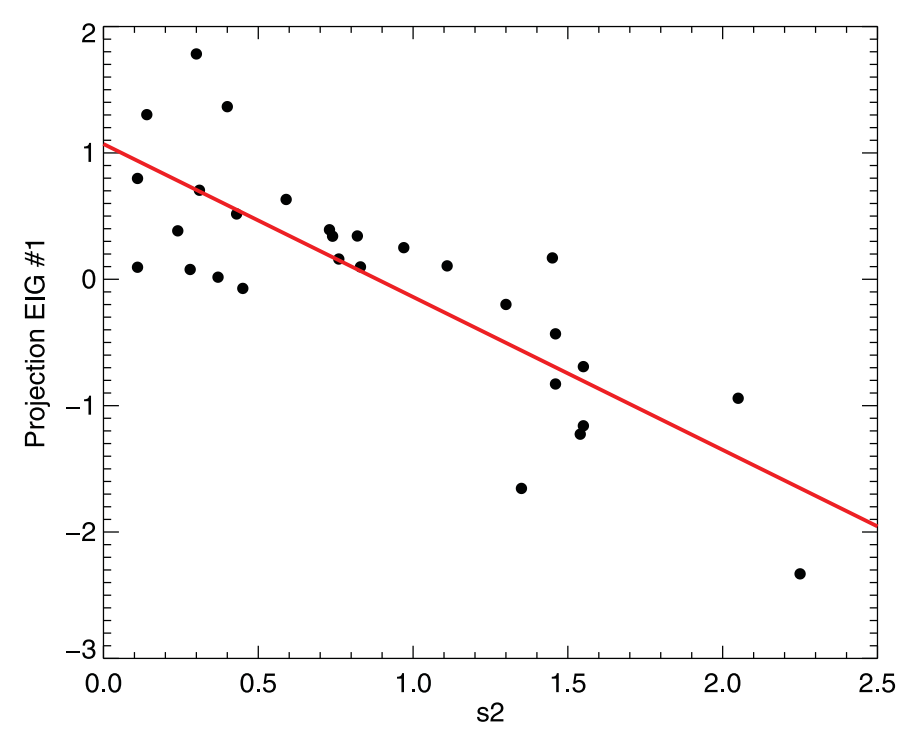

Figure 2. Correlation between the projections of the first eigenvector and the light curve parameter for which the best relation is found. Linear fit in red.

- And the fourth EV with the time elapsed between the explosion and the beginning of the plateau $\left(t_{\text {trans }}-t_{\text {exp }}\right)$, which can be understood in terms of the stretch factor for SNe Ia.

Most of these correlations have been previously found in Anderson et al. (2014), and here we have been able to reproduce them with a systematic analysis by using a statistical tool. We have also been able to reproduce the original light-curves with only these four EVs. The agreement is remarkable even with the three first components.

\section{Summary and outlook}

We presented here the results only for the $V$ band. Further analysis will be performed including other passbands. The final goal of this work is to enable the use of SNe II as standard candles only with photometric information, and to produce a Hubble diagram for type II SNe. We have compiled a sample of hundreds of spectroscopically confirmed and photometrically identified type II SNe, up to higher redshift. This sample will be analyzed using the same procedure.

Acknowledgements. Support for LG is provided by the Ministry of Economy, Development, and Tourism's Millennium Science Initiative through grant IC12009, awarded to The Millennium Institute of Astrophysics (MAS), and by CONICYT through FONDECYT grant 3140566.

\section{References}

Hamuy, M. \& Pinto, P. A 2002, ApJ, 566, 63

Anderson, J. P., González-Gaitán, S., Hamuy, M. et al. 2014, ApJ, 786, 67A 